Hans Petter Langtangen

\title{
Python Scripting \\ for Computational Science
}

Third Edition

With 62 Figures

43 Springer 


\section{Table of Contents}

1 Introduction

1.1 Scripting versus Traditional Programming

1.1.1 Why Scripting is Useful in Computational Science .... 2

1.1.2 Classification of Programming Languages .................. 4

1.1.3 Productive Pairs of Programming Languages ............. 5

1.1.4 Gluing Existing Applications …………………………..... 6

1.1.5 Scripting Yields Shorter Code ....................................... 7

1.1.6 Efficiency ........................................................................ 8

1.1.7 Type-Specification (Declaration) of Variables ............. 9

1.1.8 Flexible Function Interfaces ............................................. 11

1.1.9 Interactive Computing …………………………………... 12

1.1.10 Creating Code at Run Time ........................................... 13

1.1.11 Nested Heterogeneous Data Structures ......................... 14

1.1 .12 GUI Programming .......................................................... 16

1.1.13 Mixed Language Programming ...................................... 17

1.1.14 When to Choose a Dynamically Typed Language 19

1.1 .15 Why Python? ................................................................... 20

1.1.16 Script or Program? ........................................................... 21

1.2 Preparations for Working with This Book ……………………. 22

2 Getting Started with Python Scripting .................. 27

$2.1 \quad$ A Scientific Hello World Script .................................................... 27

2.1.1 Executing Python Scripts .............................................. 28

2.1.2 Dissection of the Scientific Hello World Script .......... 29

2.2 Working with Files and Data ....................................................... 32

2.2.1 Problem Specification ……………………..................... 32

2.2.2 The Complete Code ............................................................ 33

2.2.3 Dissection ......................................................................... 33

2.2.4 Working with Files in Memory ……………………….... 36

2.2.5 Array Computing ............................................................ 37

2.2.6 Interactive Computing and Debugging ……………...... 39

2.2.7 Efficiency Measurements ................................................. 42

2.2.8 Exercises ........................................................................... 43

2.3 Gluing Stand-Alone Applications …………………………........ 46

2.3.1 The Simulation Code ........................................................... 47

2.3.2 Using Gnuplot to Visualize Curves ............................... 49

2.3.3 Functionality of the Script ……………………….......... 50

2.3.4 The Complete Code ........................................................ 51

2.3.5 Dissection …………………………………………....... 53

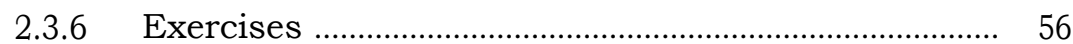

2.4 Conducting Numerical Experiments …………………............... 58

2.4.1 Wrapping a Loop Around Another Script .................. 59 
2.4.2 Generating an HTML Report ..................................... 60

2.4.3 Making Animations ....................................................... 61

2.4.4 Varying Any Parameter ................................................... 63

$2.5 \quad$ File Format Conversion ............................................................... 66

2.5.1 A Simple Read/Write Script ........................................... 66

2.5.2 Storing Data in Dictionaries and Lists ....................... 68

2.5.3 Making a Module with Functions ................................ 69

2.5.4 Exercises ................................................................. 71

3 Basic Python ................................................................ 73

3.1 Introductory Topics .................................................................... 74

3.1.1 Recommended Python Documentation ........................ 74

3.1.2 Control Statements .................................................. 75

3.1.3 Running Applications .................................................... 76

3.1.4 File Reading and Writing ............................................. 78

3.1.5 Output Formatting …...................................................... 80

3.2 Variables of Different Types ….................................................... 81

3.2.1 Boolean Types ................................................................. 82

3.2.2 The None Variable ............................................................ 82

3.2.3 Numbers and Numerical Expressions ......................... 83

3.2.4 Lists and Tuples ............................................................. 85

3.2.5 Dictionaries .............................................................. 91

3.2.6 Splitting and Joining Text ........................................... 94

3.2.7 String Operations ........................................................... 95

3.2.8 Text Processing ................................................................ 97

3.2.9 The Basics of a Python Class ........................................ 99

3.2.10 Copy and Assignment ..................................................... 101

3.2.11 Determining a Variable's Type .................................... 105

3.2.12 Exercises ................................................................... 106

3.3 Functions ............................................................................. 110

3.3.1 Keyword Arguments .................................................. 111

3.3.2 Doc Strings ..................................................................... 112

3.3.3 Variable Number of Arguments ................................... 113

3.3.4 Call by Reference ............................................................ 114

3.3.5 Treatment of Input and Output Arguments ............. 116

3.3.6 Function Objects ............................................................ 117

3.4 Working with Files and Directories ......................................... 118

3.4.1 Listing Files in a Directory .......................................... 118

3.4.2 Testing File Types ........................................................... 119

3.4.3 Removing Files and Directories ................................... 120

3.4.4 Copying and Renaming Files ........................................ 121

3.4.5 Splitting Pathnames ......................................................... 122

3.4.6 Creating and Moving to Directories ............................ 122

3.4.7 Traversing Directory Trees ............................................ 123

3.4.8 Exercises ............................................................ 125 
4 Numerical Computing in Python 131

4.1 A Quick NumPy Primer ......................................................... 132

4.1.1 Creating Arrays ……………………………………...... 132

4.1.2 Array Indexing ............................................................ 136

4.1.3 Loops over Arrays ......................................................... 138

4.1.4 Array Computations ..................................................... 139

4.1.5 More Array Functionality ................................................ 142

4.1.6 Type Testing .................................................................. 144

4.1.7 Matrix Objects ................................................................. 145

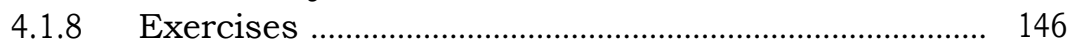

4.2 Vectorized Algorithms ................................................................. 147

4.2.1 From Scalar to Array in Function Arguments ............ 147

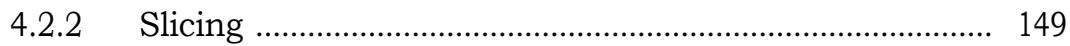

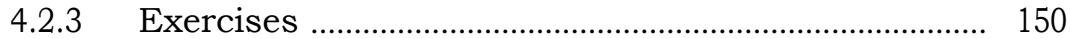

4.3 More Advanced Array Computing ……………………………...... 151

4.3.1 Random Numbers ......................................................... 152

4.3.2 Linear Algebra ……………………………………..... 153

4.3.3 Plotting …….................................................................. 154

4.3.4 Example: Curve Fitting ………………………………... 157

4.3.5 Arrays an Structured Grids .......................................... 159

4.3.6 File I/O with NumPy Arrays ……………………….... 163

4.3.7 Functionality in the Numpyutils Module .................... 166

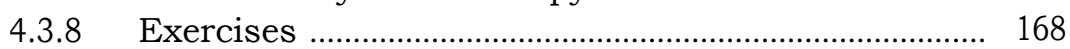

4.4 Other Tools for Numerical Computations ................................ 173

4.4.1 The ScientificPython Package ....................................... 173

4.4.2 The SciPy Package ........................................................ 178

4.4.3 The Python-Matlab Interface ....................................... 183

4.4.4 Symbolic Computing in Python ..................................... 184

4.4.5 Some Useful Python Modules .......................................... 186

5 Combining Python with Fortran, C, and C++ .... 189

5.1 About Mixed Language Programming ………………................ 189

5.1.1 Applications of Mixed Language Programming ........... 190

5.1.2 Calling C from Python .................................................. 190

5.1.3 Automatic Generation of Wrapper Code ................... 192

5.2 Scientific Hello World Examples ……………………………..... 194

5.2.1 Combining Python and Fortran ………….................... 195

5.2.2 Combining Python and C ............................................. 201

5.2.3 Combining Python and C++ Functions ..................... 208

5.2.4 Combining Python and C++ Classes ......................... 210

5.2.5 Exercises …....................................................................... 214

5.3 A Simple Computational Steering Example ............................... 215

5.3.1 Modified Time Loop for Repeated Simulations ........... 216

5.3.2 Creating a Python Interface ………………..................... 217

5.3.3 The Steering Python Script .......................................... 218

5.3.4 Equipping the Steering Script with a GUI .................. 222

5.4 Scripting Interfaces to Large Libraries ………………………..... 223 
6 Introduction to GUI Programming ............................ 227

6.1 Scientific Hello World GUI …………………………………...... 228

6.1.1 Introductory Topics ........................................................ 228

6.1.2 The First Python/Tkinter Encounter ........................... 230

6.1.3 Binding Events ................................................................... 233

6.1.4 Changing the Layout ......................................................... 234

6.1.5 The Final Scientific Hello World GUI ........................... 238

6.1.6 An Alternative to Tkinter Variables .............................. 240

6.1.7 About the Pack Command ............................................ 241

6.1.8 An Introduction to the Grid Geometry Manager 243

6.1.9 Implementing a GUI as a Class .................................... 245

6.1.10 A Simple Graphical Function Evaluator ...................... 247

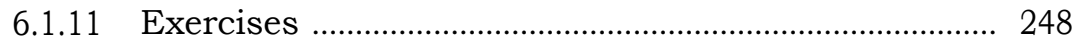

6.2 Adding GUIs to Scripts ........................................................... 250

6.2.1 A Simulation and Visualization Script with a GUI .. 250

6.2.2 Improving the Layout ................................................. 253

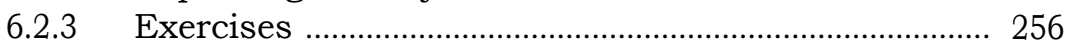

6.3 A List of Common Widget Operations ………………………... 257

6.3.1 Frame ………......................................................... 259

6.3.2 Label ....................................................................... 260

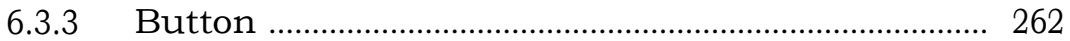

6.3.4 Text Entry …………………………………………....... 262

6.3.5 Balloon Help .............................................................. 264

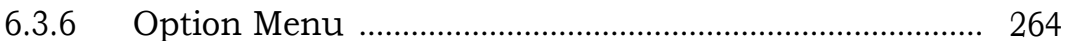

6.3.7 Slider ....................................................................... 265

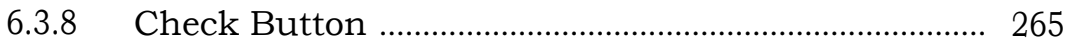

6.3.9 Making a Simple Megawidget .......................................... 266

6.3.10 Menu Bar ……………………………….................... 267

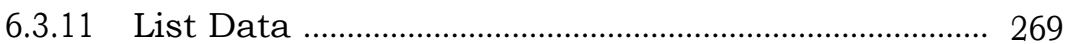

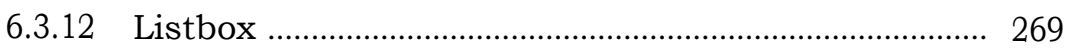

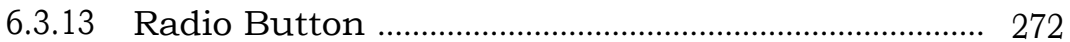

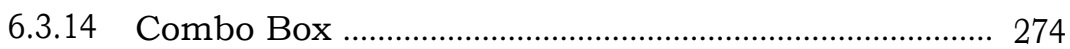

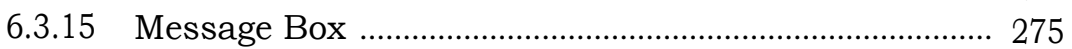

6.3.16 User-Defined Dialogs ......................................................... 276

6.3.17 Color-Picker Dialogs ........................................................ 278

6.3.18 File Selection Dialogs .................................................... 278

6.3.19 Toplevel ..................................................................... 280

6.3.20 Some Other Types of Widgets ........................................ 281

6.3.21 Adapting Widgets to the User's Resize Actions ......... 282

6.3.22 Customizing Fonts and Colors ........................................ 284

6.3.23 Widget Overview .......................................................... 286

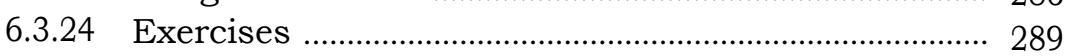


7 Web Interfaces and CGI Programming 295

7.1 Introductory CGI Scripts ……………………………………...... 296

7.1.1 Web Forms and CGI Scripts .......................................... 297

7.1.2 Generating Forms in CGI Scripts ............................... 299

7.1.3 Debugging CGI Scripts .................................................. 301

7.1.4 A General Shell Script Wrapper for CGI Scripts ... 302

7.1.5 Security Issues ............................................................... 304

7.2 Adding Web Interfaces to Scripts ................................................. 306

7.2.1 A Class for Form Parameters ……………….................. 306

7.2.2 Calling Other Programs ................................................. 308

7.2.3 Running Simulations ..................................................... 309

7.2.4 Getting a CGI Script to Work ……………................... 311

7.2.5 Using Web Applications from Scripts ........................... 313

7.2.6 Exercises ..................................................................... 316

8 Advanced Python ............................................................. 319

8.1 Miscellaneous Topics .................................................................... 319

8.1.1 Parsing Command-Line Arguments .............................. 319

8.1.2 Platform-Dependent Operations ……………………... 322

8.1.3 Run-Time Generation of Code ...................................... 323

8.1.4 Exercises ........................................................................... 324

8.2 Regular Expressions and Text Processing ……………………. 326

8.2.1 Motivation .................................................................. 326

8.2.2 Special Characters ......................................................... 329

8.2.3 Regular Expressions for Real Numbers ......................... 331

8.2.4 Using Groups to Extract Parts of a Text ................... 334

8.2.5 Extracting Interval Limits .............................................. 335

8.2.6 Extracting Multiple Matches .......................................... 339

8.2.7 Splitting Text ................................................................... 344

8.2.8 Pattern-Matching Modifiers ………………………….... 345

8.2.9 Substitution and Backreferences ................................... 347

8.2.10 Example: Swapping Arguments in Function Calls 348

8.2.11 A General Substitution Script ...................................... 351

8.2.12 Debugging Regular Expressions ……………………..... 353

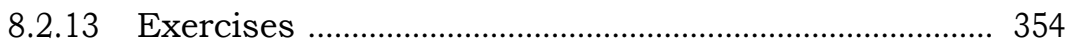

8.3 Tools for Handling Data in Files ................................................. 362

8.3.1 Writing and Reading Python Data Structures ........... 362

8.3.2 Pickling Objects ............................................................ 364

8.3.3 Shelving Objects ............................................................... 366

8.3.4 Writing and Reading Zip and Tar Archive Files ......... 366

8.3.5 Downloading Internet Files ............................................. 367

8.3.6 Binary Input/Output ……………………..................... 368

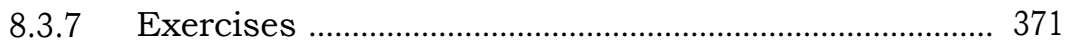

8.4 A Database for NumPy Arrays ……………………………..... 371

8.4.1 The Structure of the Database ....................................... 371

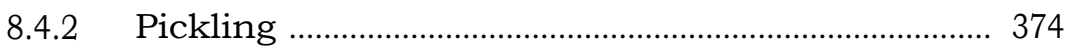

8.4.3 Formatted ASCII Storage ……………………………... 375 
8.4.4 Shelving .......................................................................... 376

8.4.5 Comparing the Various Techniques ………………....... 377

8.5 Scripts Involving Local and Remote Hosts .................................. 378

8.5.1 Secure Shell Commands ................................................. 378

8.5.2 Distributed Simulation and Visualization .................. 380

8.5.3 Client/Server Programming …………………………..... 382

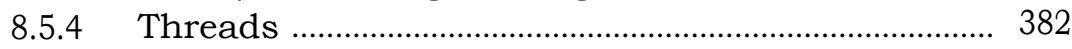

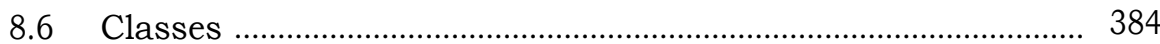

8.6.1 Class Programming ......................................................... 384

8.6.2 Checking the Class Type …………………................... 388

8.6.3 Private Data .................................................................. 389

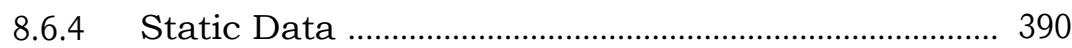

8.6.5 Special Attributes …………………………………....... 390

8.6.6 Special Methods ................................................................ 391

8.6.7 Multiple Inheritance ........................................................ 392

8.6.8 Using a Class as a C-like Structure ............................... 393

8.6.9 Attribute Access via String Names …………………... 394

8.6.10 New-Style Classes ……………………………………...... 394

8.6.11 Implementing Get/Set Functions via Properties ....... 395

8.6.12 Subclassing Built-in Types …………………………..... 396

8.6.13 Building Class Interfaces at Run Time ........................ 399

8.6.14 Building Flexible Class Interfaces …………………..... 403

8.6.15 Exercises ..................................................................... 409

8.7 Scope of Variables .......................................................................... 413

8.7.1 Global, Local, and Class Variables .............................. 413

8.7.2 Nested Functions .............................................................. 415

8.7.3 Dictionaries of Variables in Namespaces ....................... 416

8.8 Exceptions .......................................................................... 418

8.8.1 Handling Exceptions ...................................................... 419

8.8.2 Raising Exceptions .......................................................... 420

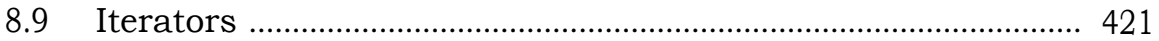

8.9.1 Constructing an Iterator ................................................... 421

8.9.2 A Pointwise Grid Iterator .................................................. 423

8.9.3 A Vectorized Grid Iterator ................................................ 427

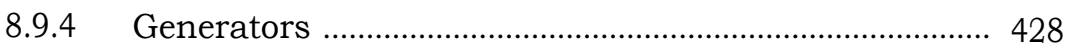

8.9.5 Some Aspects of Generic Programming ....................... 432

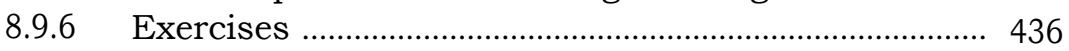

8.10 Investigating Efficiency .................................................................... 437

8.10.1 CPU-Time Measurements ............................................. 437

8.10.2 Profiling Python Scripts .................................................. 441

8.10.3 Optimization of Python Code ....................................... 442

8.10.4 Case Study an Numerical Efficiency .............................. 445 
9 Fortran Programming with NumPy Arrays .......... 451

9.1 Problem Definition ........................................................................ 451

9.2 Filling an Array in Fortran ........................................................... 453

9.2.1 The Fortran Subroutine ..................................................... 454

9.2.2 Building and Inspecting the Extension Module ........... 455

9.3 Array Storage Issues ..................................................................... 457

9.3.1 Generating an Erroneous Interface ............................... 457

9.3.2 Array Storage in C and Fortran .................................... 459

9.3.3 Input and Output Arrays as Function Arguments $\quad 459$

9.3.4 F2PY Interface Files ...................................................... 466

9.3.5 Hiding Work Arrays ........................................................... 470

9.4 Increasing Callback Efficiency ………………………………..... 470

9.4.1 Callbacks to Vectorized Python Functions ................... 471

9.4.2 Avoiding Callbacks to Python ........................................ 473

9.4.3 Compiled Inline Callback Functions ............................. 474

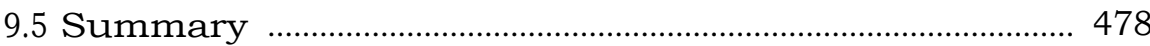

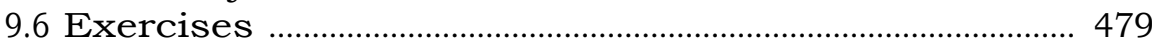

$10 \mathrm{C}$ and C++ Programming with NumPy Arrays .. 483

10.1 Automatic Interfacing of C/C++ Code ...................................... 484

10.1.1 Using F2PY ................................................................ 485

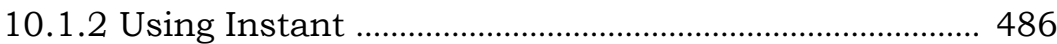

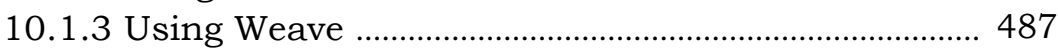

10.2 C Programming with NumPy Arrays ......................................... 488

10.2.1 The Basics of the NumPy C API ................................... 489

10.2.2 The Handwritten Extension Code .................................. 491

10.2.3 Sending Arguments from Python to C .......................... 492

10.2.4 Consistency Checks ........................................................... 493

10.2.5 Computing Array Values .................................................. 494

10.2.6 Returning an Output Array ............................................. 496

10.2.7 Convenient Macros ........................................................ 497

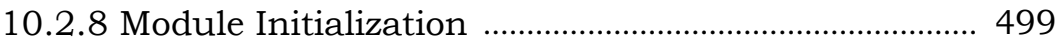

10.2.9 Extension Module Template ............................................ 500

10.2.10 Compiling, Linking, and Debugging the Module ......... 502

10.2.11 Writing a Wrapper for a C Function .............................. 503

10.3 C++ Programming with NumPy Arrays ................................... 506

10.3.1 Wrapping a NumPy Array in a C++ Object .............. 506

10.3.2 Using SCXX .................................................................... 508

10.3.3 NumPy-C++ Class Conversion ………………………... 511

10.4 Comparison of the Implementations ………………………….... 519

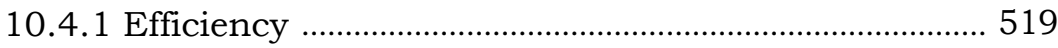

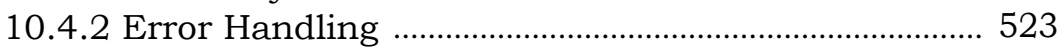

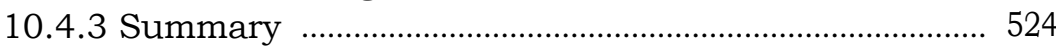

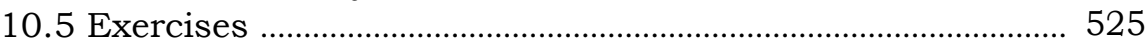


XVIII Table of Contents

11 More Advanced GUI Programming ..................... 529

11.1 Adding Plot Areas in GUIs .......................................................... 529

11.1.1 The BLT Graph Widget ................................................. 530

11.1.2 Animation of Functions in BLT Graph Widgets ......... 536

11.1.3 Other Tools for Making GUIs with Plots ...................... 538

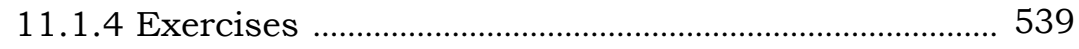

11.2 Event Bindings ............................................................................... 541

11.2.1 Binding Events to Functions with Arguments ............. 542

11.2.2 A Text Widget with Tailored Keyboard Bindings 544

11.2.3 A Fancy List Widget .......................................................... 547

11.3 Animated Graphics with Canvas Widgets ................................. 550

11.3.1 The First Canvas Encounter …………………………..... 551

11.3.2 Coordinate Systems ………………………………….... 552

11.3.3 The Mathematical Model Class ..................................... 556

11.3.4 The Planet Class ............................................................. 557

11.3.5 Drawing and Moving Planets ........................................ 559

11.3.6 Dragging Planets to New Positions ............................... 560

11.3.7 Using Pmw's Scrolled Canvas Widget ............................ 564

11.4 Simulation and Visualization Scripts ........................................... 566

11.4.1 Restructuring the Script ................................................ 567

11.4.2 Representing a Parameter by a Class ........................... 569

11.4.3 Improved Command-Line Script .................................... 583

11.4.4 Improved GUI Script ................................................... 584

11.4.5 Improved CGI Script .................................................. 585

11.4.6 Parameters with Physical Dimensions .......................... 586

11.4.7 Adding a Curve Plot Area .............................................. 588

11.4.8 Automatic Generation of Scripts ................................. 589

11.4.9 Applications of the Tools .............................................. 590

11.4.10 Allowing Physical Units in Input Files .......................... 596

11.4.11 Converting Input Files to GUIs ...................................... 601

12 Tools and Examples ........................................... 605

12.1 Running Series of Computer Experiments .................................. 605

12.1.1 Multiple Values of Input Parameters ............................ 606

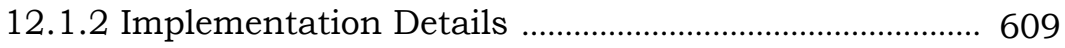

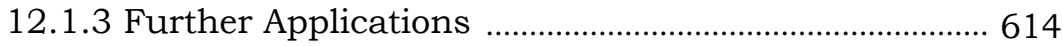

12.2 Tools for Representing Functions .................................................. 618

12.2.1 Functions Defined by String Formulas .......................... 618

12.2.2 A Unified Interface to Functions ................................... 623

12.2.3 Interactive Drawing of Functions .................................. 629

12.2.4 A Notebook for Selecting Functions ............................. 633

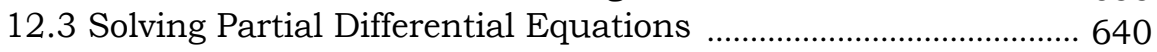

12.3.1 Numerical Methods for 1D Wave Equations ............... 641

12.3.2 Implementations of 1D Wave Equations ............................ 644

12.3.3 Classes for Solving 1D Wave Equations ......................... 651

12.3.4 A Problem Solving Environment .................................. 657

12.3.5 Numerical Methods for 2D Wave Equations .............. 663 
A Setting up the Required Software Environment .... 677

A.1 Installation on Unix Systems ...................................................... 677

A.1.1 A Suggested Directory Structure ……………………... 677

A.1.2 Setting Some Environment Variables ………………..... 678

A.1.3 Installing Tcl/Tk and Additional Modules ..................... 679

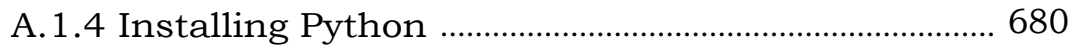

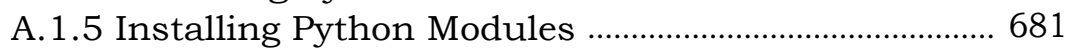

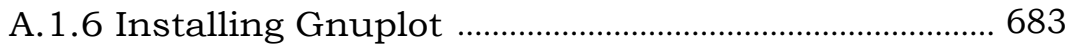

A.1.7 Installing SWIG …………………………………….... 684

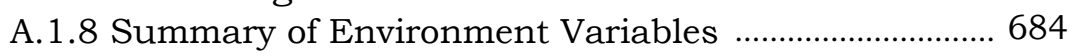

A.1.9 Testing the Installation of Scripting Utilities ……….... 685

A.2 Installation on Windows Systems ……………………………..... 685

B Elements of Software Engineering ............................. 689

B.1 Building and Using Modules ......................................................... 689

B.1.1 Single-File Modules ...................................................... 689

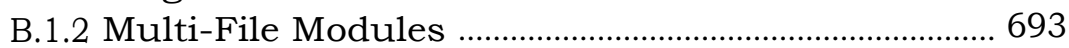

B.1.3 Debugging and Troubleshooting ……………………..... 694

B.2 Tools for Documenting Python Software …………………….... 696

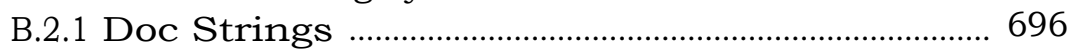

B.2.2 Tools for Automatic Documentation .............................. 698

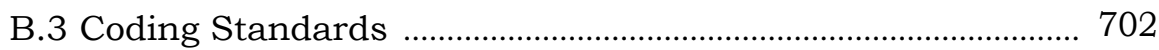

B.3.1 Style Guide .................................................................... 702

B.3.2 Pythonic Programming ………………………………....... 706

B.4 Verification of Scripts ...................................................................... 711

B.4.1 Automating Regression Tests .......................................... 711

B.4.2 Implementing a Tool for Regression Tests .................. 715

B.4.3 Writing a Test Script ..................................................... 719

B.4.4 Verifying Output from Numerical Computations ...... 720

B.4.5 Automatic Doc String Testing ……………………....... 724

B.4.6 Unit Testing ................................................................... 726

B.5 Version Control Management ………………………………..... 728

B.5.1 Mercurial _....................................................................... 729

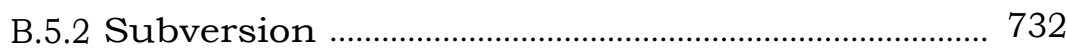

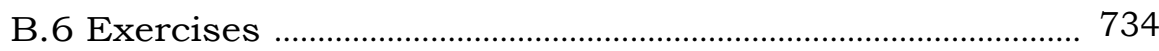

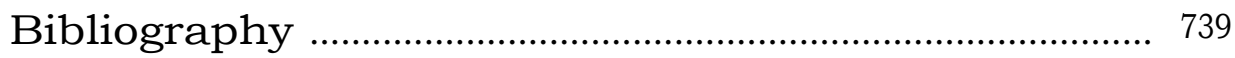

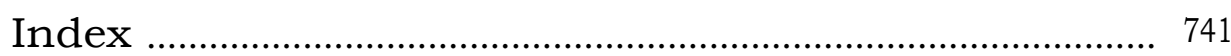

